\title{
A model for the advantage of early electron cyclotron current drive in the suppression of neoclassical tearing modes
}

\author{
Avrilios Lazaros \\ National Technical University of Athens, Greece \\ Association Euratom-Hellenic Republic \\ Marc Maraschek and Hartmut Zohm \\ Max Planck Institute for Plasma Physics, Garching Germany \\ Euratom Association
}

\begin{abstract}
An analytic model for the advantage of the early application of electron cyclotron current drive (ECCD) in the suppression of neoclassical tearing modes (NTMs) is presented. The improved performance of early ECCD is attributed to the second (smaller) saturation island width, which appears for sufficiently small (relative to the ECCD deposition width) critical island widths, in the strongly non-linear growth rate profile. The operational range for the advantage of early ECCD is obtained and it is shown that it is favored by broad deposition profiles. The preliminary experimental results in ASDEX Upgrade [H. Zohm et al., Nucl. Fusion 41, 197 (2001)] are consistent with the present model.
\end{abstract}




\section{INTRODUCTION}

Neoclassical tearing modes (NTMs) are beta-limiting instabilities, which have been observed and investigated in most high-beta tokamaks [1] [2] [3] [4]. Their behavior and control by electron cyclotron current drive (ECCD) has been also investigated in ASDEX Upgrade [5], DIII-D [6] and JT-60U [7] [8], and it was confirmed that these modes can be (partly or completely) suppressed by localized ECCD inside the magnetic island, or even avoided by early application of ECCD (i.e. before the growth of the NTM) at the rational surface [9]. A unique result, however, recently obtained in JT-60U [7] during early and late ECCD in identical discharges was that the saturation amplitude of these modes in the former case (i.e. during early ECCD) was significantly lower than in the latter case (i.e. during late ECCD). The interpretation, proposed by the JT-60U Team [8], is that the presence of a pre-existing magnetic island has an impact on the effect of localized ECCD on the equilibrium and, hence, local stability criteria (such as the equilibrium $\Delta^{\prime}$ ) are different for late and early application of ECCD (with and without a magnetic island). In the present work we present an analytic model in which the advantage of early ECCD is attributed to the non-linear growth rate of the NTM, which exhibits two stable saturation amplitudes, and the presence of the magnetic island has no impact on the equilibrium during localized ECCD. Subsequently we present preliminary experimental evidence which supports this interpretation in ASDEX Upgrade. 


\section{THE NON-LINEAR GROWTH-RATE OF NTMS}

The growth and the evolution of magnetic islands can be described by the well-known Rutherford equation, which in the presence of the neoclassical excitation and localized ECCD has the form [10]

$$
\frac{\tau_{r}}{r_{s}} \frac{d W}{d t}=r_{s} \Delta^{\prime}(W)+\alpha_{\beta}\left(\frac{r_{s} W}{W^{2}+W_{c}^{2}}-\frac{r_{s} W_{p}^{2}}{W^{3}}\right)-\alpha_{c d} F_{c d}\left(\frac{W}{\delta_{c d}}\right) .
$$

In Eq. (1):

- $W$ is the width of the magnetic island and $r_{s}$ is the radius of the rational surface (at $q=m / n$ );

- $r_{s} \Delta^{\prime}(W)$ is the classical term in the presence of (early or late) ECCD, which is usually represented by the linear expression $r_{s} \Delta^{\prime}(W)=r_{s} \Delta_{0}^{\prime}-\Delta_{1}^{\prime} W$ (the effect of ECCD on the classical term [11] [12] is implicitly included in $\Delta_{0}^{\prime}$ );

- $\alpha_{\beta}=\sqrt{r_{s} / R_{0}}\left(L_{q} / L_{p}\right) \beta_{\theta}$ (for circular poloidal cross-sections), where $L_{q} \equiv q /|\nabla q|$ is the shear length, $L_{p} \equiv-p /|\nabla p|$ is the pressure length, and $\beta_{\theta} \equiv 2 \mu_{0} p / B_{\theta}^{2}$ is the "poloidal beta";

- $W_{c}$ and $W_{p}$ are the critical island width [13] and the polarization width [14], which in mathematical terms provide the stabilization of small islands and; finally

- $\alpha_{c d}=\frac{f_{c d}}{2 \sqrt{\pi}} \frac{r_{s}}{\delta_{c d}} \frac{L_{q}}{\delta_{c d}} \frac{I_{E C C D}}{I_{P}\left(r_{s}\right)}$, where $f_{c d}$ is a fitted parameter adjacent to the profile function $F_{c d}\left(W / \delta_{c d}\right)$. 
A convenient analytic approximation [15] of the profile function $F_{c d}(w)$ (where $w \equiv W / \delta_{c d}$ and $\delta_{c d}$ is the half-width of the driven current) is

$$
F_{c d}(w)=0.3 \times w^{-1} \quad \text { for } w \leq 2 \text {, and }
$$

$F_{c d}(w)=\left[w^{2} \exp \left(w^{-1}\right)\right]^{-1} \quad$ for $w \geq 2$.

The above expression exhibits all features of the precise growth-rate profiles (involving elliptical integrals) [10], including the correct scaling with the island width in both limits of small and large islands. The adjacent (to the above profile function) parametric value of $f_{c d}=34$ is obtained from a fit of the results presented in Ref. [15]. With the definition of the normalized island widths of $w_{c} \equiv W_{c} / \delta_{c d}$ and $w_{p} \equiv W_{p} / \delta_{c d}$, Eq. (1) takes the form:

$$
\frac{d w}{d t} \propto\left(r_{s} \Delta_{0}^{\prime}-\delta_{c d} \Delta_{1}^{\prime} w\right)+\alpha_{\beta} \frac{r_{s}}{\delta_{c d}}\left(\frac{w}{w^{2}+w_{c}^{2}}-\frac{w_{p}^{2}}{w^{3}}\right)-\alpha_{c d} F_{c d}(w),
$$

and, hence,

$$
\dot{w} \propto\left(g_{0}-g_{1} w\right)+\frac{w}{w^{2}+w_{c}^{2}}-\frac{w_{p}^{2}}{w^{3}}-\alpha F_{c d}(w),
$$

where

$$
g_{0} \equiv \frac{\delta_{c d} \Delta_{0}^{\prime}}{\alpha_{\beta}}=\sqrt{\frac{R_{0}}{r_{s}}} \frac{L_{p}}{L_{q}} \frac{\delta_{c d} \Delta_{0}^{\prime}}{\beta_{\theta}},
$$

and

$$
\alpha \equiv \frac{\delta_{c d}}{r_{s}} \frac{\alpha_{c d}}{\alpha_{\beta}}=\frac{f_{c d}}{2 \sqrt{\pi}} \sqrt{\frac{R_{0}}{r_{s}}} \frac{L_{p}}{\delta_{c d}} \beta_{\theta}^{-1} \frac{I_{E C C D}}{I_{P}\left(r_{s}\right)} .
$$


Assuming that the critical island width is the dominant term in small island stability, from Eq. (2) we obtain:

$\dot{w} \propto\left(g_{0}-g_{1} w\right)+\frac{w}{w^{2}+w_{c}^{2}}-\alpha F_{c d}(w)$

Growth rate profiles, obtained by Eq. (5), for the same equilibrium (i.e. constant $g_{0}$ and $g_{1}$ ) and a variety of parametric values of $\alpha$ and $w_{c}$ are illustrated in Fig. 1. It is seen that for sufficiently small $w_{c}$ (and in the proper parametric range of $\alpha$ ) Eq.(5) exhibits two stable solutions with $\dot{w}=0$ and $d \dot{w} / d w<0$. One stable solution at $w<2$, and a second one at $w>2$ (in fact there are up to four solutions, two of which with $\dot{w}=0$ and $d \dot{w} / d w>0$ are unstable). In mathematical terms the second (smaller) stable solution is a consequence of the different scaling of the current-drive term (with the island width) for small and large islands compared to the deposition width (i.e. $F_{c d}(w) \propto w^{-1}$ for $w<2$ and $F_{c d}(w) \propto w^{-2}$ for $w>2$ ); and the same scaling of the neoclassical term for small and large islands (i.e. $\propto w^{-1}$ ) at $w_{c}=0$. The smaller solution disappears when the scaling of the neoclassical term also changes for small islands (i.e. $\propto w$ ) by introducing a finite $w_{c}$. It is also understood that in the growth rate profiles, previously obtained for ASDEX Upgrade [5] and DIII-D [6], the second stable solution did not appear because the value of $w_{c}$ which was assumed was not sufficiently small. On the contrary the smaller stable solution is clearly seen in the precise growth rate profiles in Ref. [10], in which $w_{c}$ was 
assumed to be zero. The "advantage" of the early ECCD, consequently, could be addressed by the two stable saturation amplitudes as follows:

- During early ECCD at the rational $(q=m / n)$ surface (in the absence of a magnetic island) the NTM grows when a sufficiently broad seed island develops. The growing island eventually saturates when its width reaches the smaller stable solution.

- During late ECCD, inside a pre-existing saturated island, the growthrate of the island becomes negative (i.e. $d w / d t<0$ ), and the island shrinks. The shrinking island eventually saturates when its width reaches the larger stable solution of the modified growth-rate profile.

In Fig. (1) it is also seen that for early ECCD in the absence of the second (smaller) stable solution, the growth of the NTM is much slower and requires a broader seed island, but the NTM finally saturates at the same amplitude with the late application. In different words, an advantage of early ECCD is always present in the triggering of the NTM, but not always in the saturation.

\section{THE NECESSARY CONDITIONS FOR THE ADVANTAGE OF EARLY ECCD}

For the assessment of the advantage of the early ECCD, we assume $g_{1} \approx 0$ (i.e. $\Delta^{\prime}$ constant independent of $w$ ) and Eq. (5) takes a simpler form, which involves only three parameters (i.e. $g_{0} w_{c}$ and $\alpha$ )

$\dot{w} \propto g_{0}+\frac{w}{w^{2}+w_{c}^{2}}-\alpha F_{c d}(w)$ 
As we explained in Section II the necessary and sufficient condition for the advantage of early ECCD is the presence of the two stable solutions with $\dot{w}=0$ and $d \dot{w} / d w<0$ (one at $w>2$, and a second one at $w<2$ ). The approach to the problem in this formal manner involves third order equations which couple all of the three parameters and (although still possible) is not suited to give physical insight. In our present approach to the problem we examine the necessary conditions in which the constrains on $\alpha$ and $g_{0}$ can be separated and compared to the experiment.

One necessary condition for the two stable solutions, which becomes obvious in Fig. 1, is that the growth rate profile has a minimum at $w=2$. In mathematical terms this equivalent to

$$
\begin{aligned}
& {[d \dot{w} / d w]_{(w=2-)}<0} \\
& {[d \dot{w} / d w]_{(w=2+)}>0}
\end{aligned}
$$

The advantage of Eqs. (7) is that the derivative (with respect to $w$ on both sides) eliminates the constant parameter $g_{0}$, and couples only $\alpha$ and $w_{c}$. Using in Eq. (6) the different analytic expressions of $F_{c d}(w)$ (for $w<2$ and $w>2$ ), Eqs. (7) subsequently take the form

$$
\begin{aligned}
& \frac{d}{d w}\left[\frac{w}{w^{2}+w_{c}^{2}}-\frac{0.3 \alpha}{w}\right]_{(w=2)}<0 \\
& \frac{d}{d w}\left[\frac{w}{w^{2}+w_{c}^{2}}-\frac{\alpha}{w^{2} \exp \left(w^{-1}\right)}\right]_{(w=2)}>0
\end{aligned}
$$

and, hence, 
$\alpha<13 \times \frac{4-w_{c}^{2}}{\left(4+w_{c}^{2}\right)^{2}}$

$8.8 \times \frac{4-w_{c}^{2}}{\left(4+w_{c}^{2}\right)^{2}}<\alpha$

Equations (9a) and (9b) determine the necessary upper and lower limits of $\alpha$ (in terms of $w_{c}$ ) for the advantage of early ECCD. The parametric range of $\alpha$, in which the two domains overlap, is shown in Fig. 2 together with examples of growth rate profiles in each domain.

A second necessary condition for the presence of the smaller stable solution, which also becomes obvious in Fig. 1, is that the minimum value of the growth-rate at $w=2$ is negative, and the maximum value of the growth rate for $w<2$ is positive, i.e.

$$
\begin{aligned}
& \dot{w}(w=2)<0 \\
& \dot{w}_{\max }(w<2)>0
\end{aligned}
$$

Equation (10a), from Eq. (6), takes the form

$$
\left[g_{0}+\frac{w}{w^{2}+w_{c}^{2}}-\frac{0.3 \alpha}{w}\right]_{(w=2)}<0 \text { and, hence, } g_{0}<\frac{0.3 \alpha}{2}-\frac{2}{4+w_{c}^{2}}
$$

Using subsequently Eq. (9a) we obtain 
$g_{0}<\frac{0.3}{2} 13 \frac{4-w_{c}^{2}}{\left(4+w_{c}^{2}\right)^{2}}-\frac{2}{4+w_{c}^{2}}$

The maximum value of $\dot{w}$ is obtained from Eq. (6) for $\alpha=0$ and $d \dot{w} / d w=0$, which is satisfied at $w=w_{c}$ and, hence, $\dot{w}_{\max }=g_{0}+1 / 2 w_{c}$. From Eq. (10b), subsequently, we obtain

$-1 / 2 w_{c}<g_{0}$

Equations (11a) and (11b) determine the necessary upper and lower limits of $g_{0}$ (in terms of $w_{c}$ ) for the advantage of early ECCD. The parametric range of $g_{0}$, in which the two domains overlap, is shown in Fig. 3 together with examples of growth rate profiles in each domain. The parametric ranges of $\alpha$ and $g_{0}$ (in terms of $w_{c}$ ), for the advantage of early ECCD, are illustrated together in Fig. (4). It is seen that the broadest range of values is obtained for $w_{c}<0.3$, and that both parametric ranges disappear at $w_{c}=2$, i.e. when the critical island width becomes equal to the deposition width.

\section{APPLICATION TO ASDEX UPGRADE}

Figure 4 can be useful for comparisons with the experiment only if it is transformed to provide the operational range of the controlled parameters (such as the deposition half-width $\delta_{c d}$ and the driven current $I_{E C C D}$ ) rather than the range of the parameters $\alpha, g_{0}$. From the definitions in Eqs. (3) and (4) we obtain 


$$
\begin{aligned}
& \frac{I_{E C C D}}{I_{P}\left(r_{s}\right)}=\frac{2 \sqrt{\pi}}{f_{c d}} \sqrt{\frac{r_{s}}{R_{0}}} \frac{\delta_{c d}}{L_{p}} \beta_{\theta} \alpha \\
& \Delta_{0}^{\prime}=\delta_{c d}^{-1} \sqrt{\frac{r_{s}}{R_{0}}} \frac{L_{q}}{L_{p}} \beta_{\theta} g_{0}
\end{aligned}
$$

Keeping also in mind that $\alpha=\alpha\left(W_{c} / \delta_{c d}\right)$ and $g_{0}=g_{0}\left(W_{c} / \delta_{c d}\right)$, as regime boundaries in Fig. 4 are functions of the normalized critical island width, the boundaries of the operational range of $I_{E C C D}$ and the equilibrium range of $\Delta_{0}^{\prime}$ (for the advantage of early ECCD) can be obtained (from Fig. 4) as functions of $\delta_{c d}$, for a certain value of $W_{c}$. With the estimated value of $W_{c}=1.8 \mathrm{~cm}$ [5], and the typical values of the high-beta plasmas of ASDEX Upgrade, i.e. $R_{0}=170 \mathrm{~cm}, \quad a=39 \mathrm{~cm}, \quad r_{s}=23 \mathrm{~cm}, \quad L_{p} \approx 10 \mathrm{~cm}$, $L_{q} \approx 31 \mathrm{~cm}, \beta_{\theta}=2.4$, the operational range of $I_{E C C D}$ and the equilibrium range of $\Delta_{0}^{\prime}$ (for the advantage of early ECCD) are illustrated in Fig. 5. The "flat" lower limit of $\Delta_{0}^{\prime}$ essentially is trivial and simply indicates that for "more negative" values the NTM would be stable. The upper limit, however, is essential, especially for narrow deposition half-widths. For $\Delta_{0}^{\prime}$ inside the colored range in Fig.5, the three operational regimes of $I_{E C C D}$ correspond to the complete suppression of the NTM, the partial suppression of the NTM with the advantage of early ECCD, and the partial suppression of the NTM without the advantage of early ECCD. For $\Delta_{0}^{\prime}$ outside the colored range in Fig.5, the second operational regime of $I_{E C C D}$ (which correspond to the partial suppression of the NTM with the advantage of early ECCD) simply disappears. 
Preliminary experiments on NTM control by early and late ECCD were conducted in ASDEX Upgrade at the reduced ECRH power of $0.7 M W$ (to avoid the complete suppression of the NTM), and the toroidal injection angle of $\phi_{\text {tor }}=-19^{0}$. This translates to a deposition half-width of $\delta_{e c}=3.8 \mathrm{~cm}$ and a driven current of $I_{E C C D}=11 \mathrm{kA}=0.025 \times I_{p}\left(r_{s}\right)$. The operational point of the driven current for these discharges is indicated in Fig. 5, while the equilibrium $\Delta_{0}^{\prime}$ is evaluated (and included in Fig. 5) only to the order of magnitude, using the very rough approximation of $\Delta_{0}^{\prime} \approx-2 \mathrm{~m} / r_{\mathrm{s}}=-0.26 / \mathrm{cm}$ in the absence of ECCD. As we explained above in this operational point of the driven current we should expect the partial suppression of the NTM without the advantage by early ECCD in the saturation amplitude. The behavior of the $m / n=3 / 2$ NTM in these discharges is shown in Fig. 6. It is actually seen that the $m / n=3 / 2$ NTM in early ECCD grows with a significant delay (compared to the late), but saturates at the same amplitude as expected.

\section{CONCLUSIONS}

An analytic theoretical model for the advantage of early ECCD in the suppression of NTMs was presented, in which the improved performance of early ECCD is attributed to the second (smaller) saturation width, which appears for sufficiently small ratio of the critical island width to the ECCD deposition width, in the strongly non-linear growth rate profile. The preliminary experiment in ASDEX Upgrade has confirmed the advantage of the early ECCD in the triggering of the NTM, but not in its saturation because the operational point was not in the proper range. A systematic experimental investigation of this effect, (including power scans and 
deposition scans) is foreseen in ASDEX Upgrade, which should confirm the advantage of early ECCD in the proper operational regime of Fig. 5.

Although the preliminary results in Fig. 6 did not confirm the advantage of early ECCD in the suppression of the NTM, they provide a very important information. The saturation of the NTM precisely at the same amplitude, in early and late ECCD, in Fig. 6 indicates that in ASDEX Upgrade the presence of the island had no impact on the equilibrium, which was the interpretation proposed by the JT60 group. In fact the three operational regimes of Fig. 5, which also allow the saturation of the NTM at the same amplitude in early and late ECCD, are relevant only to the present interpretation. In the model based on the impact of the island on the equilibrium, the saturation amplitude of the NTM should be always smaller in early ECCD.

\section{ACKNOWLEDGEMENTS}

The present work was carried out within the framework of the European Fusion Programme, during a Euratom mobility of A.L. at IPP Garching. The views and opinions expressed above do not necessarily reflect those of Euratom. 


\section{REFERENCES}

[1] Z. Chang, J.D. Callen, E.D. Fredrickson, et al., Phys. Rev. Lett. 74, 4663 (1995)

[2] R.J. La Haye, L.L. Lao, E.J. Strait, et al., Nucl. Fusion 37, 397 (1997)

[3] H. Zohm, D.A. Gates, H.R. Wilson, et al., Plasm. Phys. Contr. Fusion. 39, B237 (1997)

[4] JET Team, Nucl. Fusion 39, 1965 (1999)

[5] H. Zohm, G. Gantenbein, A. Gude, et al., Phys. Plasmas 8, 2009 (2001)

[6] R.J. La Haye, S. Guenter, D.A. Humpreys, et al., Phys. Plasmas 9, 2051 (2002)

[7] K. Nagasaki, A. Isayama, S. Ide, et al., Nucl. Fusion 43, L7 (2003)

[8] K. Nagasaki, A. Isayama, N. Hayashi, et al., Nucl. Fusion 45, 1608 (2005)

[9] R.J. La Haye, D.A. Humpreys, J.R. Ferron, et al., Nucl. Fusion 45, L37 (2005)

[10] C.C. Hegna and J.D. Callen, Phys. Plasmas 4, 2940 (1997)

[11] E. Westerhof, Nucl. Fusion 30, 1143 (1990)

[12] A. Pletzer and F.W. Perkins, Phys. Plasmas 6, 1589 (1999)

[13] R. Fitzpatrick, Phys. Plasmas 2, 825 (1995)

[14] H.R. Wilson, J.W. Connor, R.J. Hastie, et al., Phys. Plasmas 3, 248 (1996)

[15] A.W. Morris, P.G. Carolan, R. Fitzpatrick et al., Phys. Fluids B 4, 413 (1992) 


\section{FIGURE CAPTIONS}

Figure 1. Simulated growth-rate profiles of NTMs in the presence of ECCD, at the same equilibrium (with $g_{0}=-8 \times 10^{-2}, g_{1}=2 \times 10^{-3}$ ), and the parametric values of $w_{c}=0.0,0.1,1.0$ and $\alpha=0,2,3,4$. The saturation width in each profile is marked by a cross $(\times)$, while the second (smaller) stable solution, whenever exists, is marked by a circle.

Figure 2. Illustration of the parametric range of $\alpha$, in which the two necessary conditions of Eqs. (9a) and (9b) overlap, together with examples of growth rate profiles in each domain. The corresponding profiles and domains are indicated by the same markers (square, triangle, and circle).

Figure 3. Illustration of the parametric range of $g_{0}$, in which the two necessary conditions of Eqs. (11a) and (11b) overlap, together with examples of growth rate profiles in each domain. The corresponding profiles and domains are indicated the same markers (square, triangle, and circle). 
Figure 4. Illustration of the parametric ranges of $\alpha$ and $g_{0}$ (in terms of $w_{c}$ ), for a mode behavior as follows:

(1a) complete suppression by ECCD;

(1b) partial suppression with a possible advantage of early ECCD;

(1c) partial suppression without the advantage of early ECCD;

(2a) partial suppression without the advantage of early ECCD;

(2b) partial suppression with a possible advantage of early ECCD;

(2c) stable mode;

Figure 5. Illustration of the operational ranges of $I_{E C C D}$ and $\Delta_{0}^{\prime}$ (in terms of $\delta_{c d}$ ), for a mode behavior in ASDEX Upgrade as follows:

(1a) complete suppression by ECCD;

(1b) partial suppression with a possible advantage of early ECCD;

(1c) partial suppression without the advantage of early ECCD;

(2a) partial suppression without the advantage of early ECCD;

(2b) partial suppression with a possible advantage of early ECCD;

(2c) stable mode;

The triangular marker indicates the operational point for the discharges in Fig. 6.

Figure 6. The variation of the amplitude of the $m / n=3 / 2$ NTM, during early and late ECCD (0.7MW at toroidal injection angle of 19 degrees) in identical discharges of ASDEX Upgrade. 


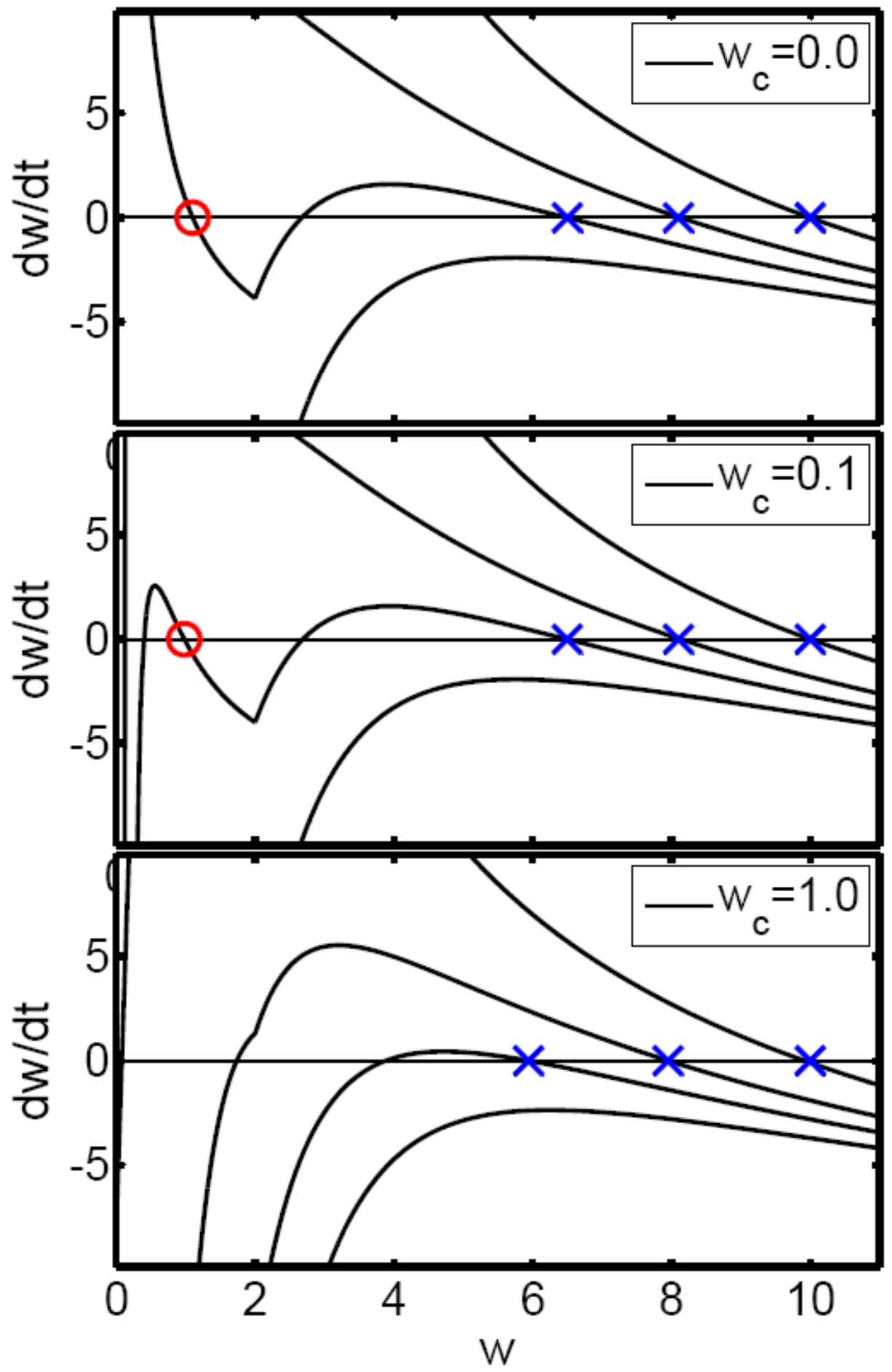

Figure 1. 

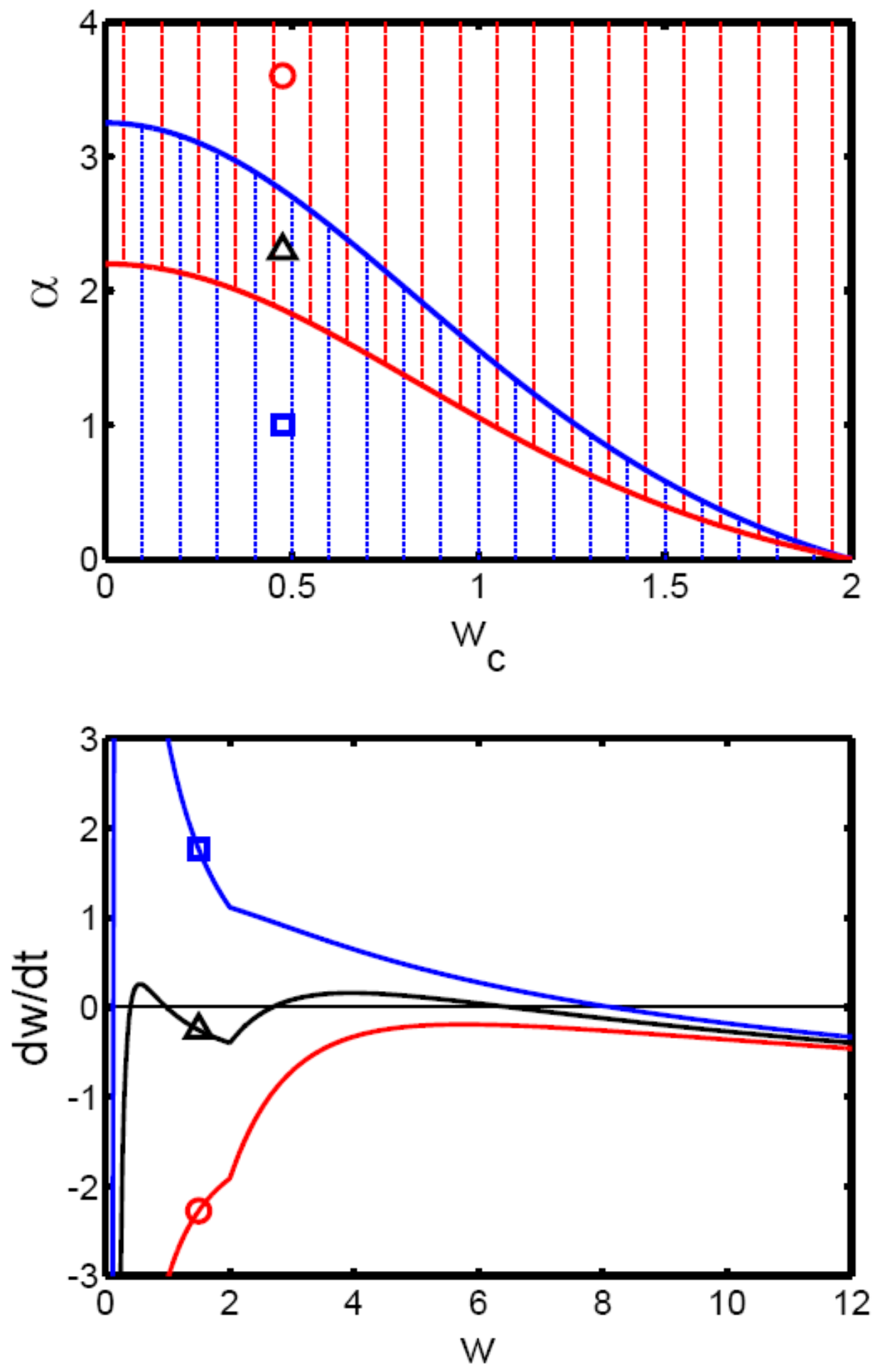

Figure 2. 

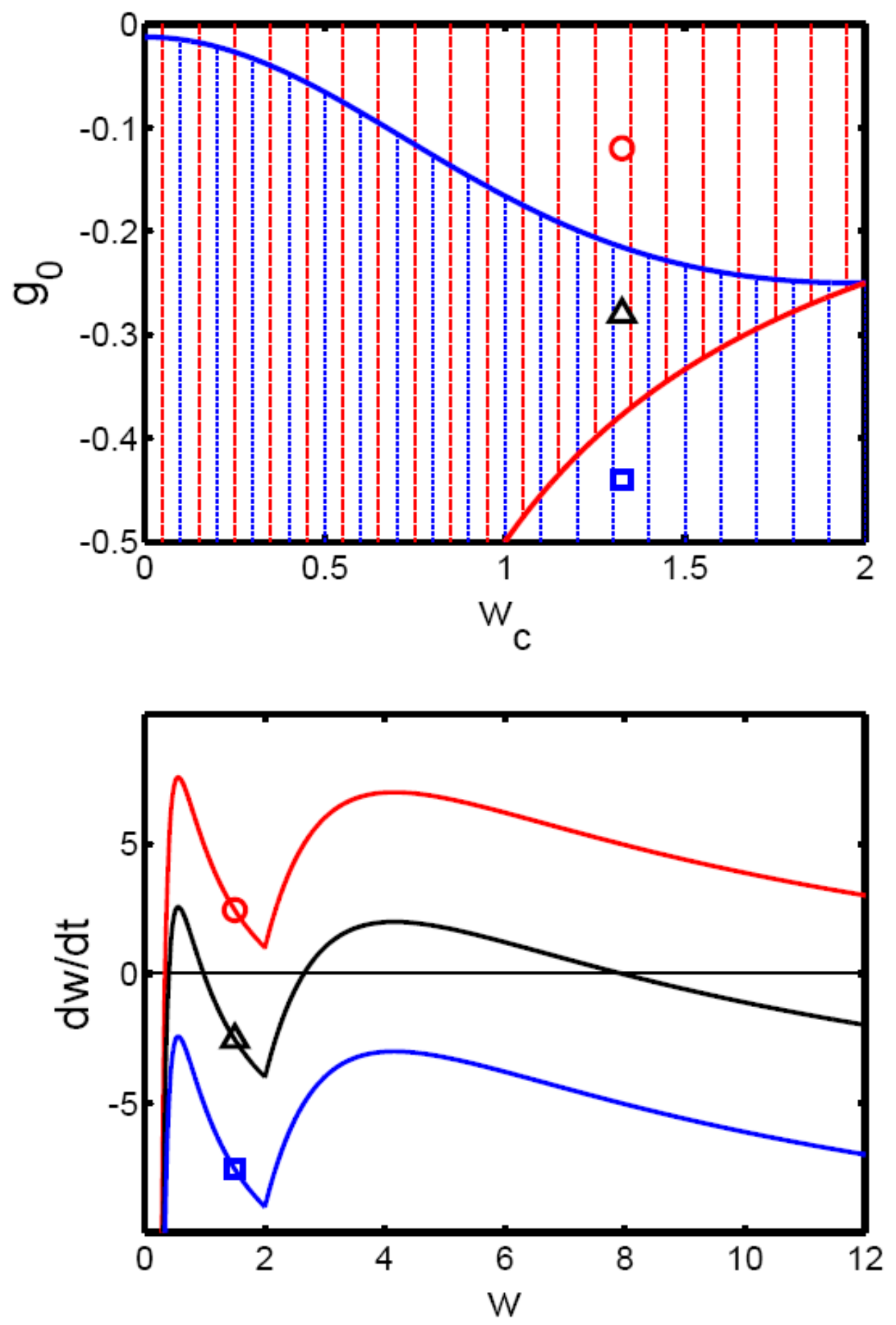

Figure 3. 


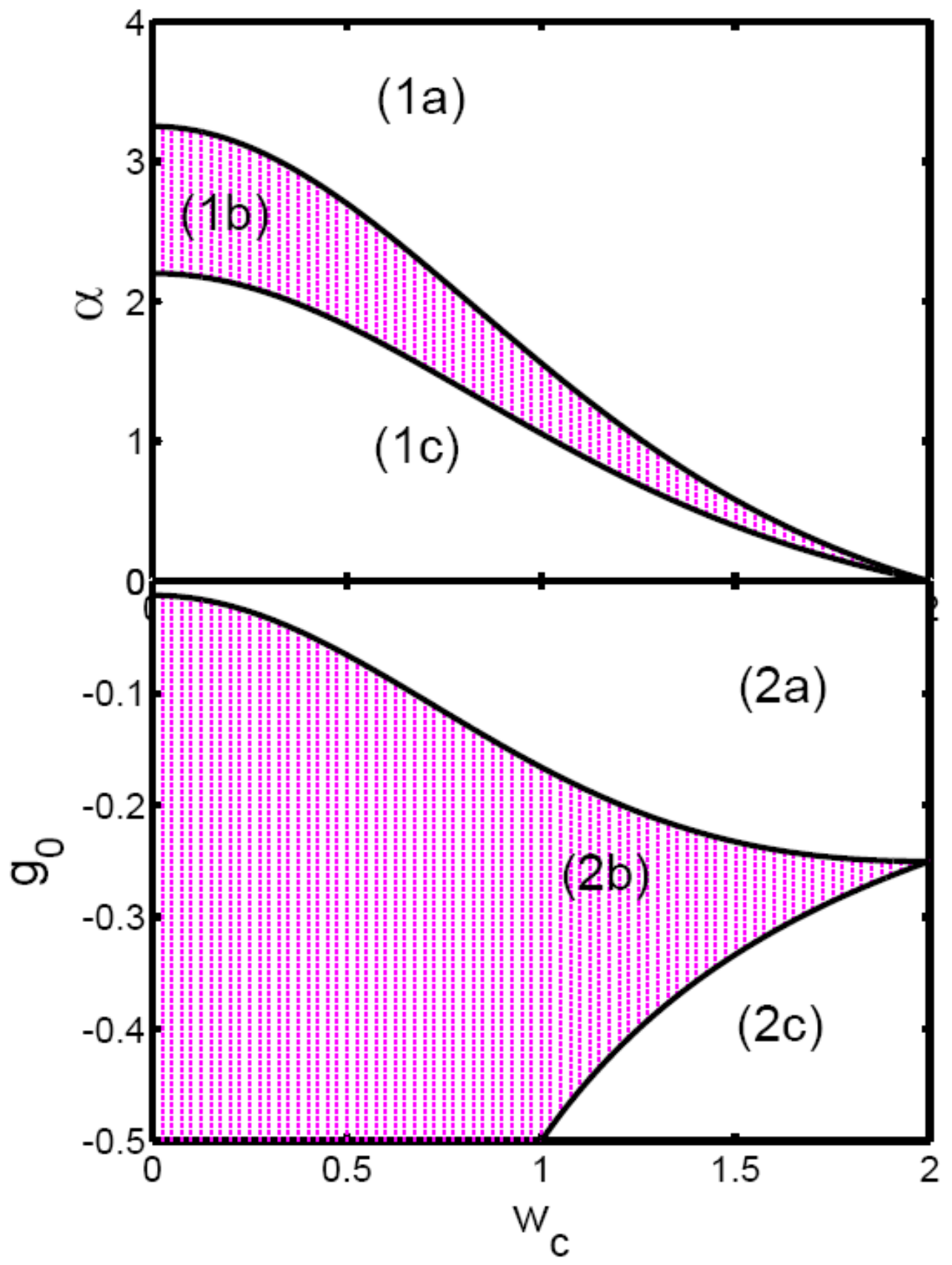

Figure 4. 


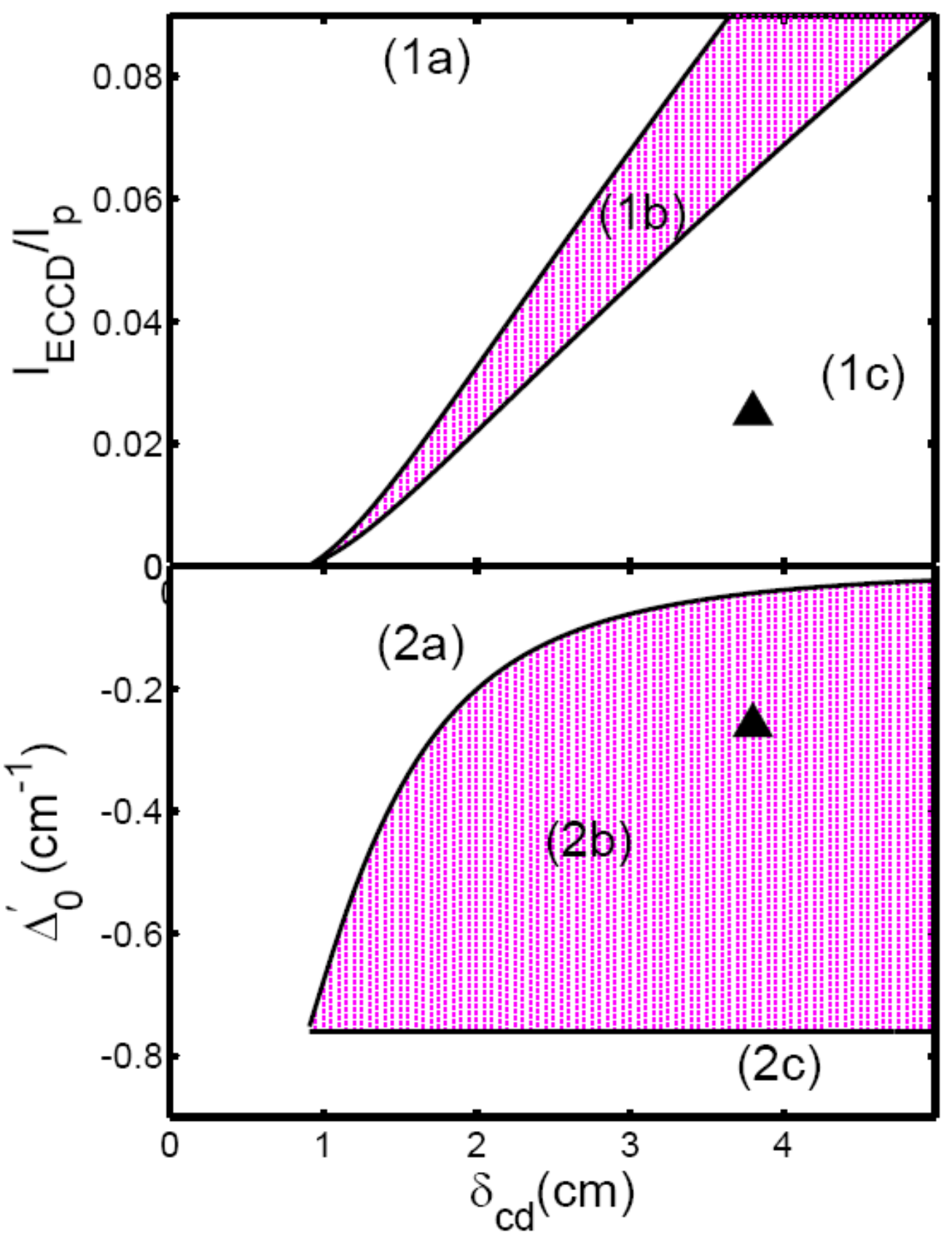

Figure 5. 

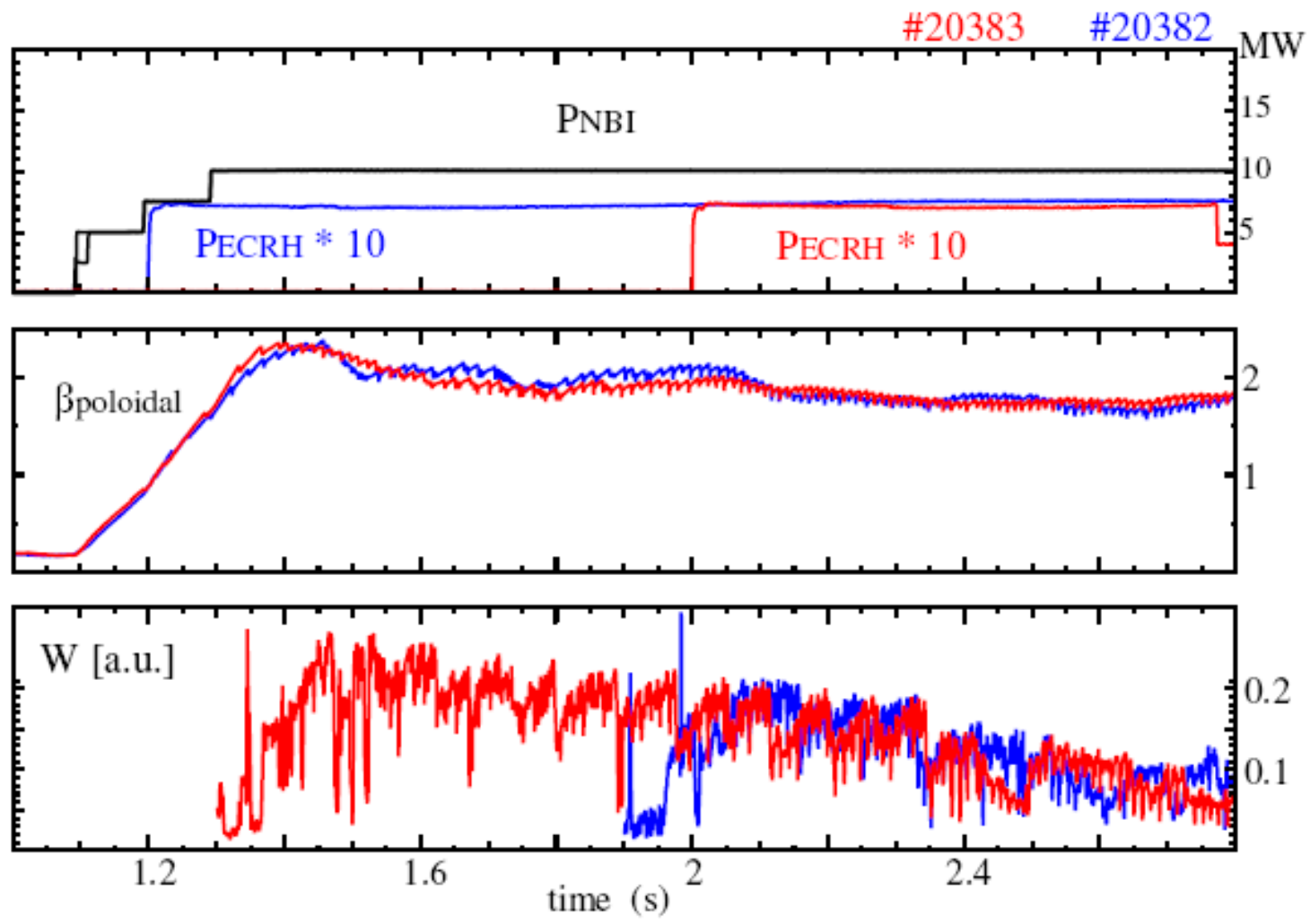

Figure 6. 